\title{
ON CLASSES OF ANALYTIC FUNCTIONS CONTAINING GENERALIZATION OF INTEGRAL OPERATOR
}

\author{
Maslina Darus ${ }^{1, *}$ AND RabHa W. IBrahim ${ }^{2}$ \\ ${ }^{1}$ School of Mathematical Sciences, Faculty of Science and Technology, \\ Universiti Kebangsaan Malaysia Bangi 43600, Selangor Darul Ehsan, \\ Malaysia, maslina@ukm.my \\ ${ }^{2}$ School of Mathematical Sciences, Faculty of Science and Technology, \\ Universiti Kebangsaan Malaysia Bangi 43600, Selangor Darul Ehsan, \\ Malaysia, rabhaibrahim@yahoo.com
}

\begin{abstract}
New classes containing generalization of integral operator are introduced. Characterization and other properties of these classes are investigated. Further, Fekete-Szegö functional for these classes are also given.

Key words and Phrases: Integral operator, analytic functions, differential operator, Fekete-Szegö functional, distortion theorem.

Abstrak. Pada paper ini diperkenalkan kelas baru yang memuat perumuman dari operator integral. Kemudian karakterisasi dan sifat lain juga dikaji dari kelas. Lebih lanjut, fungsional Fekete-Szegö untuk kelas ini juga diberikan.

Kata kunci: Perumuman operator integral, fungsi analitik, operator diferensial, fungsional Fekete-Szegö, teorema distorsi.
\end{abstract}

\section{Introduction}

Let $\mathcal{H}$ be the class of functions analytic in $U$ and $\mathcal{H}[a, n]$ be the subclass of $\mathcal{H}$ consisting of functions of the form $f(z)=a+a_{n} z^{n}+a_{n+1} z^{n+1}+\ldots$. Let $\mathcal{A}$ be the subclass of $\mathcal{H}$ consisting of functions of the form

$$
f(z)=z+\sum_{n=2}^{\infty} a_{n} z^{n}, \quad z \in U
$$

2000 Mathematics Subject Classification: 30C 45.

Received: 17-12-2010, revised: 07-04-2011, accepted: 09-04-2011.

*Corresponding author 
Now we introduce a differential operator defined as follows : $\mathbf{D}_{\lambda, \delta}^{k, \alpha, \beta}: \mathcal{A} \rightarrow \mathcal{A}$ by

$$
\mathbf{D}_{\lambda, \delta}^{k, \alpha, \beta} f(z)=z+\sum_{n=2}^{\infty}\left[(\beta n)^{\alpha}+(n-1)(\beta n)^{\alpha} \lambda\right]^{k} C(\delta, n) a_{n} z^{n}
$$

where $k \in \mathbb{N}_{0}, \alpha \geq 0, \lambda \geq 0, \delta \geq 0$,

and

$$
C(\delta, n)=\left(\begin{array}{c}
n+\delta-1 \\
\delta
\end{array}\right)=\frac{\Gamma(n+\delta)}{\Gamma(n) \Gamma(\delta+1)}
$$

Remark 1.1. When $\alpha=\beta=1, \lambda=0, \delta=0$ or $\alpha=0, \lambda=1, \delta=0$ we get Sălăgean differential operator [18], $k=0$ gives Ruschewyh operator [17], $\alpha=0, \delta=0$ implies Al-Oboudi differential operator of order (k) $[1], \alpha=\beta=1, \lambda=0$ or $\alpha=0, \lambda=1$ operator (2) reduces to Al-shaqsi and Darus differential operator of order $(\mathrm{k})[2]$ and $\alpha=0$ poses the differential operator of order $(\mathrm{k})$, which is given by the authors [3]. Note that the differential operator in [3] was initially introduced by Al-Shaqsi and Darus [20]. However, it was overlooked and restated again in [3]. Recently, the authors posed different kind of linear, differential and integral operators (see $[4-13,19])$.

Given two functions $f, g \in \mathcal{A}, \quad f(z)=z+\sum_{n=2}^{\infty} a_{n} z^{n}$ and $g(z)=z+\sum_{n=2}^{\infty} b_{n} z^{n}$ their convolution or Hadamard product $f(z) * g(z)$ is defined by

$$
f(z) * g(z)=z+\sum_{n=2}^{\infty} a_{n} b_{n} z^{n}, \quad z \in U .
$$

And for several functions $f_{1}(z), \ldots, f_{m}(z) \in \mathcal{A}$

$$
f_{1}(z) * \ldots * f_{m}(z)=z+\sum_{n=2}^{\infty}\left(a_{1 n} \ldots a_{m n}\right) z^{n}, \quad, z \in U .
$$

Analogous to $\mathbf{D}_{\lambda, \delta}^{k, \alpha, \beta} f(z), z \in U$ we define an integral operator $\mathbf{J}_{\lambda, \delta}^{k, \alpha}: \mathcal{A} \rightarrow \mathcal{A}$ as follows.

Let

$$
\begin{gathered}
\phi(z):=\frac{z}{1-z}+\frac{\lambda z}{(1-z)^{2}}-\frac{\lambda z}{1-z}, \lambda \geq 0 \\
F_{k}(z)=\underbrace{\phi(z) * \ldots * \phi(z)}_{k-\text { times }} * \underbrace{\frac{z \beta}{(1-z)^{2}} * \ldots * \frac{z \beta}{(1-z)^{2}}}_{\alpha k-\text { times }} *\left[\frac{z}{(1-z)^{\delta+1}}\right] \\
=z+\sum_{n=2}^{\infty}\left[(\beta n)^{\alpha}+(n-1)(\beta n)^{\alpha} \lambda\right]^{k} C(\delta, n) z^{n}
\end{gathered}
$$

where $k, \alpha \in \mathbb{N}_{0}, \beta \geq 1, \delta \geq 0, \lambda \geq 0$. 
And let $F_{k}^{(-1)}$ be defined such that

$$
\begin{aligned}
F_{k}(z) * F_{k}^{(-1)} & =\frac{z}{1-z} \\
& =z+\sum_{n=2}^{\infty} z^{n} .
\end{aligned}
$$

Note that $F_{k}^{(-1)}$ is needed to get the integral operator from differential operator.

Then

$$
\begin{aligned}
\mathbf{J}_{\lambda, \delta}^{k, \alpha, \beta} f(z) & =F_{k}^{(-1)} * f(z) \\
& =[\underbrace{\phi(z) * \ldots * \phi(z)}_{k-\text { times }} * \underbrace{\frac{z \beta}{(1-z)^{2}} * \ldots * \frac{z \beta}{(1-z)^{2}}}_{\alpha k-\text { times }} * \frac{z}{(1-z)^{\delta+1}}]^{(-1)} * f(z) \\
& =z+\sum_{n=2}^{\infty} \frac{a_{n}}{\left[(\beta n)^{\alpha}+(n-1)(\beta n)^{\alpha} \lambda\right]^{k} C(\delta, n)} z^{n},
\end{aligned}
$$

where $k, \alpha \in \mathbb{N}_{0}, \beta \geq 1, \lambda \geq 0, \delta \geq 0 \quad z \in U$.

Remark 1.2. When $\alpha=0, \lambda=1, \delta=0$ we get an integral operator (see [18]), $k=0$ gives Noor integral operator $[15,16]$.

Some of relations for this integral operator are discussed in the next lemma.

Lemma 1.1. Let $f \in \mathcal{A}$. Then

(i) $\mathbf{J}_{\lambda, 0}^{0, \alpha, \beta} f(z)=f(z)$,

(ii) $\mathbf{J}_{1,0}^{1,0, \beta} f(z)=\int_{0}^{z} \frac{f(t)}{t} d t=f(z) *(-\log (1-z))=z+\sum_{n=2}^{\infty} a_{n} z^{n} \in \mathcal{A}$.

Note that (ii) is the type of Bernardi integral[21].

Definition 1.1. Let $f(z) \in \mathcal{A}$. Then $f(z) \in \mathbf{S}_{\lambda, \delta}^{k, \alpha, \beta}(\mu)$ if and only if

$$
\Re\left\{\frac{z\left[\mathbf{J}_{\lambda, \delta}^{k, \alpha, \beta} f(z)\right]^{\prime}}{\mathbf{J}_{\lambda, \delta}^{k, \alpha, \beta} f(z)}\right\}>\mu, \quad 0 \leq \mu<1, \quad z \in U .
$$

Definition 1.2. Let $f(z) \in \mathcal{A}$. Then $f(z) \in \mathbf{C}_{\lambda, \delta}^{k, \alpha, \beta}(\mu)$ if and only if

$$
\Re\left\{\frac{\left[z\left(\mathbf{J}_{\lambda, \delta}^{k, \alpha, \beta} f(z)\right)^{\prime}\right]^{\prime}}{\left(\mathbf{J}_{\lambda, \delta}^{k, \alpha, \beta} f(z)\right)^{\prime}}\right\}>\mu, \quad 0 \leq \mu<1, \quad z \in U .
$$

The article is organized as follows: In section 2, we study the characterization and distortion theorems, and other properties of these classes. In section 3 , we obtain 
sharp upper bound of $\left|a_{2}\right|$ and of the Fekete-Szegö functional $\left|a_{3}-\nu a_{2}^{2}\right|$ for the classes $S_{\lambda, \delta}^{k, \alpha, \beta}(\mu)$ and $C_{\lambda, \delta}^{k, \alpha, \beta}(\mu)$. For this purpose we need the following result

Lemma 1.2.[14] Let $p \in \mathcal{P}$, that is, $p$ be analytic in $U$, be given by $p(z)=1+$ $\sum_{n=1}^{\infty} p_{n} z^{n}$ and $\Re\{p(z)\}>0$ for $z \in U$. Then

$$
\left|p_{2}-\frac{p_{1}^{2}}{2}\right| \leq 2-\frac{\left|p_{1}\right|^{2}}{2}
$$

and $\left|p_{n}\right| \leq 2$ for all $n \in N$.

\section{General Properties of $\mathbf{J}_{\lambda, \delta}^{k, \alpha, \beta}$}

In this section we study the characterization properties and distortion theorems for the function $f(z) \in \mathcal{A}$ to belong to the classes $\mathbf{S}_{\lambda, \delta}^{k, \alpha, \beta}(\mu)$ and $\mathbf{C}_{\lambda, \delta}^{k, \alpha, \beta}(\mu)$ by obtaining the coefficient bounds.

Theorem 2.1. Let $f(z) \in \mathcal{A}$. If for $\alpha \geq 0, \beta \geq 1, \delta \geq 0$ and $\lambda \geq 0$

$$
\sum_{n=2}^{\infty} \frac{(n-\mu)\left|a_{n}\right|}{\left[(\beta n)^{\alpha}+(n-1)(\beta n)^{\alpha} \lambda\right]^{k} C(\delta, n)} \leq 1-\mu, \quad 0 \leq \mu<1,
$$

then $f(z) \in \mathbf{S}_{\lambda, \delta}^{k, \alpha, \beta}(\mu)$. The result (4) is sharp.

Proof. Suppose that (4) holds. Since

$$
\begin{aligned}
1-\mu \geq & \sum_{n=2}^{\infty}(n-\mu) \frac{\left|a_{n}\right|}{\left[(\beta n)^{\alpha}+(n-1)(\beta n)^{\alpha} \lambda\right]^{k} C(\delta, n)} \\
\geq & \sum_{n=2}^{\infty} \mu \frac{\left|a_{n}\right|}{\left[(\beta n)^{\alpha}+(n-1)(\beta n)^{\alpha} \lambda\right]^{k} C(\delta, n)} \\
& -\sum_{n=2}^{\infty} \frac{n\left|a_{n}\right|}{\left[(\beta n)^{\alpha}+(n-1)(\beta n)^{\alpha} \lambda\right]^{k} C(\delta, n)}
\end{aligned}
$$

then this implies that

$$
\frac{1+\sum_{n=2}^{\infty} \frac{n\left|a_{n}\right|}{\left[(\beta n)^{\alpha}+(n-1)(\beta n)^{\alpha} \lambda\right]^{k} C(\delta, n)}}{\mid+\sum_{n=2}^{\infty} \frac{\left|a_{n}\right|}{\left[(\beta n)^{\alpha}+(n-1)(\beta n)^{\alpha} \lambda\right]^{k} C(\delta, n)}}>\mu,
$$

hence

$$
\Re\left\{\frac{z\left[\mathbf{J}_{\lambda, \delta}^{k, \alpha, \beta} f(z)\right]^{\prime}}{\mathbf{J}_{\lambda, \delta}^{k, \alpha, \beta} f(z)}\right\}>\mu .
$$

We also note that the assertion (4) is sharp and the extremal function is given by

$$
f(z)=z+\sum_{n=2}^{\infty} \frac{(1-\mu)}{(n-\mu)}\left[(\beta n)^{\alpha}+(n-1)(\beta n)^{\alpha} \lambda\right]^{k} C(\delta, n) z^{n} .
$$


In the same way we can verify the following results

Theorem 2.2. Let $f(z) \in \mathcal{A}$. If for $\alpha \geq 0, \beta \geq 1, \delta \geq 0$ and $\lambda \geq 0$

$$
\sum_{n=2}^{\infty} \frac{n(n-\mu)\left|a_{n}\right|}{\left[(\beta n)^{\alpha}+(n-1)(\beta n)^{\alpha} \lambda\right]^{k} C(\delta, n)} \leq 1-\mu, \quad 0 \leq \mu<1
$$

then $f(z) \in \mathbf{C}_{\lambda, \delta}^{k, \alpha, \beta}(\mu)$. The result (8) is sharp.

Theorem 2.3. Let the hypotheses of Theorem 2.1 be satisfy. Then for $z \in U$ and $0 \leq \mu<1$

$$
\left|\mathbf{J}_{\lambda, \delta}^{k, \alpha, \beta} f(z)\right| \geq|z|-\frac{1-\mu}{2-\mu}
$$

and

$$
\left|\mathbf{J}_{\lambda, \delta}^{k, \alpha, \beta} f(z)\right| \leq|z|+\frac{1-\mu}{2-\mu}
$$

Proof. By using Theorem 2.1, one can verify that

$$
\begin{aligned}
(2-\mu) & \sum_{n=2}^{\infty} \frac{\left|a_{n}\right|}{\left[(\beta n)^{\alpha}+(n-1)(\beta n)^{\alpha} \lambda\right]^{k} C(\delta, n)} \\
& \leq \sum_{n=2}^{\infty} \frac{(n-\mu)\left|a_{n}\right|}{\left[(\beta n)^{\alpha}+(n-1)(\beta n)^{\alpha} \lambda\right]^{k} C(\delta, n)} \\
& \leq 1-\mu
\end{aligned}
$$

then

$$
\sum_{n=2}^{\infty} \frac{\left|a_{n}\right|}{\left[(\beta n)^{\alpha}+(n-1)(\beta n)^{\alpha} \lambda\right]^{k} C(\delta, n)} \leq \frac{1-\mu}{2-\mu}
$$

Thus we obtain

$$
\begin{aligned}
\left|\mathbf{J}_{\lambda, \delta}^{k, \alpha, \beta} f(z)\right| & \leq|z|+\sum_{n=2}^{\infty} \frac{\left|a_{n}\right|}{\left[(\beta n)^{\alpha}+(n-1)(\beta n)^{\alpha} \lambda\right]^{k} C(\delta, n)}|z|^{n} \\
& \leq|z|+\sum_{n=2}^{\infty} \frac{\left|a_{n}\right|}{\left[(\beta n)^{\alpha}+(n-1)(\beta n)^{\alpha} \lambda\right]^{k} C(\delta, n)}|z|^{2} \\
& \leq|z|+\left[\frac{1-\mu}{2-\mu}\right]|z|^{2}
\end{aligned}
$$


The other assertion can be proved as follows

$$
\begin{aligned}
\left|\mathbf{J}_{\lambda, \delta}^{k, \alpha, \beta} f(z)\right| & =\left|z+\sum_{n=2}^{\infty} \frac{a_{n}}{\left[(\beta n)^{\alpha}+(n-1)(\beta n)^{\alpha} \lambda\right]^{k} C(\delta, n)} z^{n}\right| \\
& \geq\left|z-\sum_{n=2}^{\infty} \frac{a_{n}}{\left[(\beta n)^{\alpha}+(n-1)(\beta n)^{\alpha} \lambda\right]^{k} C(\delta, n)} z^{n}\right| \\
& \geq|z|-\sum_{n=2}^{\infty} \frac{\left|a_{n}\right|}{\left[(\beta n)^{\alpha}+(n-1)(\beta n)^{\alpha} \lambda\right]^{k} C(\delta, n)}|z|^{n} \\
& \geq|z|-\sum_{n=2}^{\infty}(n-\mu)\left[(\beta n)^{\alpha}+(n-1)(\beta n)^{\alpha} \lambda\right]^{k} C(\delta, n)\left|a_{n}\right||z|^{2} \\
& \geq|z|-\left[\frac{1-\mu}{2-\mu}\right]|z|^{2}
\end{aligned}
$$

This complete the proof.

Theorem 2.4. Let the hypotheses of Theorem 2.21 be satisfy. Then for $z \in U$ and $0 \leq \mu<1$

$$
\left|\mathbf{J}_{\lambda, \delta}^{k, \alpha, \beta} f(z)\right| \geq|z|-\frac{(1-\mu)}{2(2-\mu)}|z|^{2}
$$

and

$$
\left|\mathbf{J}_{\lambda, \delta}^{k, \alpha, \beta} f(z)\right| \leq|z|+\frac{(1-\mu)}{2(2-\mu)}|z|^{2}
$$

Theorem 2.5. Let the hypotheses of Theorem 2.1 be satisfy. Then

$$
\frac{(n-\mu)}{\left[(\beta n)^{\alpha}+(n-1)(\beta n)^{\alpha} \lambda\right]^{k} C(\delta, n)} \geq 1, \forall n \geq 2 \text { and } 0 \leq \mu<1
$$

implies

$$
|f(z)| \geq|z|-(1-\mu)|z|^{2}
$$

and

$$
|f(z)| \leq|z|+(1-\mu)|z|^{2} .
$$

Proof. In virtue of Theorem 2.1, we have

$$
\sum_{n=2}^{\infty}\left|a_{n}\right| \leq \sum_{n=2}^{\infty} \frac{(n-\mu)\left|a_{n}\right|}{\left[(\beta n)^{\alpha}+(n-1)(\beta n)^{\alpha} \lambda\right]^{k} C(\delta, n)} \leq(1-\mu)
$$

then

$$
\sum_{n=2}^{\infty}\left|a_{n}\right| \leq(1-\mu)
$$


Thus we obtain

$$
\begin{aligned}
|f(z)| & =\left|z+\sum_{n=2}^{\infty} a_{n} z^{n}\right| \\
& \leq|z|+\sum_{n=2}^{\infty}\left|a_{n}\right||z|^{2} \\
& \leq|z|+(1-\mu)|z|^{2}
\end{aligned}
$$

The other assertion can be proved as follows

$$
\begin{aligned}
|f(z)| & \geq\left|z-\sum_{n=2}^{\infty} a_{n} z^{n}\right| \\
& \geq|z|-\sum_{n=2}^{\infty}\left|a_{n}\right||z|^{2} \\
& \geq|z|-(1-\mu)|z|^{2} .
\end{aligned}
$$

This complete the proof.

Theorem 2.6. Let the hypotheses of Theorem 2.2 be satisfy. Then

$$
\frac{(n-\mu)}{\left[(\beta n)^{\alpha}+(n-1)(\beta n)^{\alpha} \lambda\right]^{k} C(\delta, n)} \geq 1, \forall n \geq 2 \text { and } 0 \leq \mu<1
$$

poses

$$
|f(z)| \geq|z|-\frac{(1-\mu)}{2}|z|^{2}
$$

and

$$
|f(z)| \leq|z|+\frac{(1-\mu)}{2}|z|^{2}
$$

\section{Fekete-Szegö for the Classes $\mathbf{S}_{\lambda, \delta}^{k, \alpha, \beta}(\mu)$ and $\mathbf{C}_{\lambda, \delta}^{k, \alpha, \beta}(\mu)$}

In this section we determine the sharp upper bound for $\left|a_{2}\right|$ for the classes $\mathbf{S}_{\lambda, \delta}^{k, \alpha, \beta}(\mu)$ and $\mathbf{C}_{\lambda, \delta}^{k, \alpha, \beta}(\mu)$. Moreover, we calculate the Fekete-Szegö $\left|a_{3}-\nu a_{2}^{2}\right|$ functional for them.

Theorem 3.1. Let the hypotheses of Theorem 2.1 be satisfy. Then

$$
\left|a_{2}\right| \leq \frac{2(1-\mu)\left[(\beta 2)^{\alpha}(1+\lambda)\right]^{k} C(\delta, 2)}{1+\mu}
$$

and for all $\nu \in \mathbb{C}$ the following bound is sharp

$$
\begin{aligned}
\left|a_{3}-\nu a_{2}^{2}\right| \leq & 2(1-\mu)\left[(3 \beta)^{\alpha}(1+2 \lambda)\right]^{k} C(\delta, 3) \\
& \max \left\{1, \mid 1+\frac{2(1-\mu)}{(1+\mu)\left[(3 \beta)^{\alpha}(1+2 \lambda)\right]^{k} C(\delta, 3)}\right. \\
& \left.-2 \nu \frac{1-\mu}{(1+\mu)^{2}} \frac{\left\{\left[(2 \beta)^{\alpha}(1+\lambda)\right]^{k} C(\delta, 2)\right\}^{2}}{\left[(3 \beta)^{\alpha}(1+2 \lambda)\right]^{k} C(\delta, 3)} \mid\right\} .
\end{aligned}
$$


Proof. Since $f \in \mathbf{S}_{\lambda, \delta}^{k, \alpha, \beta}(\mu)$ then the condition

$$
\Re\left\{\frac{z\left[\mathbf{J}_{\lambda, \delta}^{k, \alpha, \beta} f(z)\right]^{\prime}}{\mathbf{J}_{\lambda, \delta}^{k, \alpha, \beta} f(z)}\right\}>\mu, \quad 0 \leq \mu<1, \quad z \in U
$$

is equivalent to

$$
z\left[\mathbf{J}_{\lambda, \delta}^{k, \alpha, \beta} f(z)\right]^{\prime}=(1-\mu) p(z) \mathbf{J}_{\lambda, \delta}^{k, \alpha, \beta} f(z), \quad z \in U
$$

for some $p \in \mathcal{P}$. Equating coefficients we obtain $a_{2}=\frac{(1-\mu) A p_{1}}{1+\mu}, a_{3}=(1-\mu) B\left(p_{2}+\right.$ $\left.\frac{(1-\mu)}{2+\mu} p_{1}^{2}\right)$ where $A:=\left[(2 \beta)^{\alpha}(1+\lambda)\right]^{k} C(\delta, 2), B:=\left[(3 \beta)^{\alpha}(1+2 \lambda)\right]^{k} C(\delta, 3)$ and further, for $C:=\frac{(1-\mu)^{2}}{1+\mu}+\frac{(1-\mu) B}{2}-\nu\left(\frac{(1-\mu) A}{1+\mu}\right)^{2}$ and by using Lemma 1.2 we have $\left|a_{3}-\nu a_{2}^{2}\right| \leq H(x)=2(1-\mu) B+\left(C-\frac{(1-\mu) B}{2}\right) x^{2}, x:=\left|p_{1}\right|$. Consequently, we receive

$$
\left|a_{3}-\nu a_{2}^{2}\right|=\left\{\begin{array}{l}
H(0)=2(1-\mu) B, \quad C \leq \frac{(1-\mu) B}{2} \\
H(2)=4 C, \quad C>\frac{(1-\mu) B}{2} .
\end{array}\right.
$$

Equality is attained for functions given by

$$
\frac{z\left[\mathbf{J}_{\lambda, \delta}^{k, \alpha, \beta} f(z)\right]^{\prime}}{\mathbf{J}_{\lambda, \delta}^{k, \alpha, \beta} f(z)}=\frac{1+z^{2}(1-2 \mu)}{1-z^{2}}
$$

and

$$
\frac{z\left[\mathbf{J}_{\lambda, \delta}^{k, \alpha, \beta} f(z)\right]^{\prime}}{\mathbf{J}_{\lambda, \delta}^{k, \alpha, \beta} f(z)}=\frac{1+z(1-2 \mu)}{1-z}
$$

respectively.

For $\mu=0$ we receive the following corollary.

Corollary 3.1. Let the assumptions of Theorem 3.1 hold. Then for $\mu=0$

$$
\left|a_{2}\right| \leq 2\left[(2 \beta)^{\alpha}(1+\lambda)\right]^{k} C(\delta, 2)
$$

and

$$
\begin{aligned}
\left|a_{3}-\nu a_{2}^{2}\right| \leq & 2\left[(3 \beta)^{\alpha}(1+2 \lambda)\right]^{k} C(\delta, 3) \\
& \max \left\{1, \mid 1+\frac{2}{\left[(3 \beta)^{\alpha}(1+2 \lambda)\right]^{k} C(\delta, 3)}\right. \\
& \left.-2 \nu \frac{\left\{\left[(2 \beta)^{\alpha}(1+\lambda)\right]^{k} C(\delta, 2)\right\}^{2}}{\left[(3 \beta)^{\alpha}(1+2 \lambda)\right]^{k} C(\delta, 3)} \mid\right\} .
\end{aligned}
$$

In the similar manner we can prove the following result.

Theorem 3.2. Let the hypotheses of Theorem 2.2 be satisfy. then

$$
\left|a_{2}\right| \leq \frac{2(1-\mu)\left[(2 \beta)^{\alpha}(1+\lambda)\right]^{k} C(\delta, 2)}{3+\mu}
$$


and for all $\nu \in \mathbb{C}$ the following bound is sharp

$$
\begin{aligned}
\left|a_{3}-\nu a_{2}^{2}\right| & \leq \frac{2(1-\mu)}{3(2+\mu)}\left[(3 \beta)^{\alpha}(1+2 \lambda)\right]^{k} C(\delta, 3) \\
& \max \left\{1, \mid \frac{1}{2}+\frac{2(1-\mu)}{(3+\mu)}\right. \\
& \left.-\frac{3 \nu(1-\mu)}{(3+\mu)^{2}}\left\{\left[(2 \beta)^{\alpha}(1+\lambda)\right]^{k} C(\delta, 2)\right\}^{2} \mid\right\} .
\end{aligned}
$$

For $\mu=0$ we receive the following corollary.

Corollary 3.2. Let the assumptions of Theorem 3.2 hold. Then for $\mu=0$

$$
\left|a_{2}\right| \leq \frac{2}{3}\left[(2 \beta)^{\alpha}(1+\lambda)\right]^{k} C(\delta, 2)
$$

and

$\left|a_{3}-\nu a_{2}^{2}\right| \leq \frac{1}{3}\left[(3 \beta)^{\alpha}(1+2 \lambda)\right]^{k} C(\delta, 3) \max \left\{1,\left|\frac{1}{2}+\frac{2}{3}-\frac{\nu}{3}\left\{\left[(2 \beta)^{\alpha}(1+\lambda)\right]^{k} C(\delta, 2)\right\}^{2}\right|\right\}$.

Acknowledgement: The work here is fully supported by MOHE: UKM-ST-06FRGS0244-2010.

\section{References}

[1] Al-Oboudi, F., "On univalent functions defined by a generalized Salagean operator", I.J.M.M.S, 27 (2004), 1429-1436.

[2] Al-Shaqsi, K. and Darus, M., "An operator defined by convolution involving polylogarithms functions", Journal of Mathematics and Statistics, 4 (2008), 46-50.

[3] Darus, M. and Ibrahim, R.W., "Generalization of differential operator", Journal of Mathematics and Statistics, 4 (2008), 138-144.

[4] Darus, M. and Ibrahim, R.W., "On subclasses of uniformly Bazilevic type functions involving generalised differential and integral operators", Far East Journal of Mathematical Sciences (FJMS), 33 (2009), 401-411.

[5] Darus, M. and Ibrahim, R.W., "On subclasses for generalized operators of complex order", Far East Journal of Mathematical Sciences (FJMS), 33 (2009), 299-308.

[6] Darus, M. and Ibrahim, R.W., "On inclusion properties of generalized integral operator involving Noor integral", Far East Journal of Mathematical Sciences (FJMS), 33 (2009), 309-321.

[7] Darus, M. and Ibrahim, R.W., "Ibrahim and Iqbal H. Jebril, Bounded turning for generalized integral operator", Int. J. Open Problems Complex Analysis, 1 ( 2009), 1-10.

[8] Darus, M. and Ibrahim, R.W., "New Classes Containing Generalization of Differential Operator", Applied Mathematical Sciences, 3:51 (2009), 2507-2515.

[9] Darus, M. and Ibrahim, R.W., "Integral operator defined by $k$-th Hadamard product", ITB J. Sci., 42:2 (2010), 135-152.

[10] Darus, M. and Ibrahim, R.W. , "On differential operator of multivalent functions", Inequality Theory and Applications, NOVA Publishers, 6 (2010). 
[11] Darus, M. and Ibrahim, R.W., "Multivalent functions based on linear integral operator", Miskolc N.N, 11:1 (2010), 43-52.

[12] Darus, M. and Ibrahim, R.W., "Operator defined by convolution with Zeta functions", Far East Journal of Mathematical Sciences, 40:1 (2010), 93-105.

[13] Darus, M. and Ibrahim, R.W., "On univalent functions defined by a generalized differential operator", J. Anal. Appl., 16:2 (2010), 305-313.

[14] Duren, P.L., Univalent functions, Springer-Verlag, New York, 1983.

[15] Noor, K.I., "On new classes of integral operators", J. Nat. Geomet., 16 (1999), 71-80.

[16] Noor, K.I. and Noor, M.A., "On integral operators", J. of Math. Anal. Appl., 238 (1999), 341-352.

[17] Ruscheweyh, S., "New criteria for univalent functions", Proc. Amer. Math. Soc., 49 (1975), 109-115.

[18] Sălăgean, G. S., "Subclasses of univalent functions", Lecture Notes in Math., 1013, SpringerVerlag, Berlin, 1983, 362-372.

[19] Srivastava, H. M., Darus, M., and Ibrahim, R.W., "Classes of analytic functions with fractional powers defined by means of a certain linear operator", Integ. Tranc. Special Funct., 22 (2011), 17-28.

[20] Darus M. and Al-Shaqsi K., "Differential sandwich theorem with generalised derivative operator", Int. J. Comput. Math. Sci., 2:2 (2008), 75-78.

[21] Bernardi, S.D., "Convex and starlike univalent functions", Trans. Amer. Math. Soc., 135 (1969), 429-446. 